\title{
Prelog Lecture 2005
}

\author{
Eidgenössische Technische Hochschule Zürich \\ Laboratorium für Organische Chemie
}

\begin{abstract}
On Monday, November 7, 2005, the rector Prof. Dr. K. Osterwalder presented the Prelog Medal 2005 to Prof. Dr. Ben L. Feringa, University of Groningen, The Netherlands. The title of the lecture that followed was 'In Control of Chirality, from Asymmetric Catalysis to Nanomachines'.
\end{abstract}

Keywords: Feringa, B.L. · Prelog Lecture

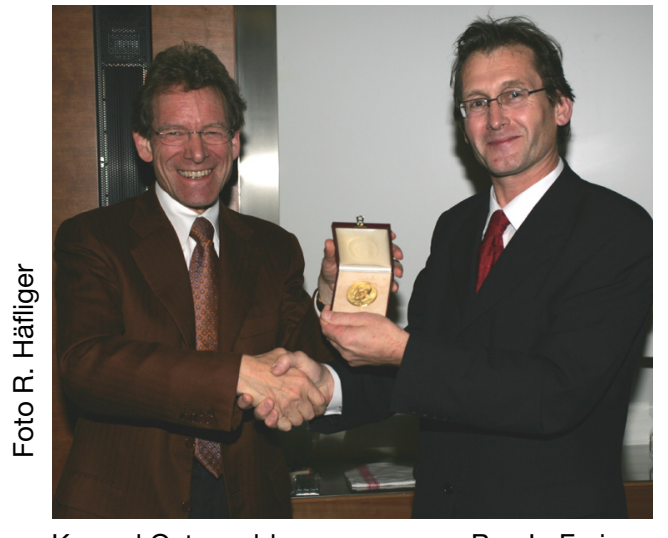

Konrad Osterwalder

Ben L. Feringa

The recipient of the 2005 Prelog Medal, Prof. Ben L. Feringa, was born in The Netherlands. He received his doctoral degree in 1978 at the University of Groningen, after working under the supervision of Prof. Hans Wynberg in the area of phenol oxidation. He subsequently took a position as a research scientist with Royal Dutch Shell, both at the Shell Research Center in Amsterdam and at the Shell Biosciences Laboratories in Sittingbourne, UK from 1978 through 1984. He joined the Department of Chemistry at the University of Groningen as a lecturer in 1984 and rapidly rose through the ranks to the position of full professor four years later as successor to Prof. Wynberg. In 2003, he was appointed as the distinguished Jacobus van't Hoff Professor of Molecular Sciences. Prof. Feringa has authored more than 300 publications and 16 patents. He is the recipient of numerous awards including the 1997 Pino Gold Metal of the Italian Chemical Society and the Spinoza award, the highest scientific award in The Netherlands. He is also an honorary member of the American Academy of Arts and Sciences.
His research interests span the broad range of cutting-edge topics in modern molecular science and include homogenous catalysis for organic synthesis, analytics, as well as nanosystems and materials (molecular switches, motors, and self-assembly). Many important, high impact insights have emanated from his research program. Recently, for example, he discovered a method for the catalytic, enantioselective conjugate addition of Grignard reagents to acceptors. This had been a fundamental and difficult problem in chemical synthesis, which had remained unsolved despite intense investigations. Additionally, he discovered the Monophos ligands, which provide an elegant, simple ligand scaffold for the generation of diverse donor libraries. The ligands have proven themselves to be useful in a host of critical processes. Moreover, these ligands have inaugurated a revolution in the field of asymmetric catalysis with transition metal complexes, which for historical reasons had come to rely in large part on the use of bisphosphine ligands. Due to his unique experience in industry and academics he tackles problems of both fundamental importance and practical relevance. Hand in hand with discoveries in reaction chemistry, he has pioneered the use of analytical methods for the rapid determination of product enantiomeric purity for use in the screening and optimization of catalytic asymmetric transformations. In the field of nanochemistry, he has developed chiral optical switches and molecular devices. The recent development of the first light-driven unidirectional molecular motors represents a particularly exciting advance. Professor Feringa's research program thus exemplifies how the coupling of a deep-seated understanding of molecular properties with masterful orchestration in chemical synthesis can lead to discovery and exploration in uncharted arenas. In doing so he has defined basic principles for further advances in this nascent, fertile area.

His broad interests in the chemical sciences are underpinned by the common theme of stereochemistry. It is thus fitting that in recognition of his scientific leadership Professor Feringa has been chosen as the recipient of 2005 Prelog Medal.

\section{Former Prelog Lecturers}

\section{Kurt Mislow}

1987 Meier Lahav and Leslie Leiserowitz

\section{K. Barry Sharpless}

1989 Jeremy R. Knowles

1990 Henri B. Kagan

1991 Clayton H. Heathcock

1992 J. Michael McBride

1993 Hisashi Yamamoto

1994 Jean-Pierre Sauvage

1995 Yoshito Kishi

1996 David M.J. Lilley

1997 Günter Helmchen

1998 Lia Addadi

1999 David Evans

2000 Helmut Schwarz

2001 Robert H. Grubbs

2002 David E. Cane

2003 Andreas Pfaltz

2004 Marvin H. Caruthers 


\title{
In Control of Chirality, from Asymmetric Catalysis to Nanomachines
}

\author{
Ben L. Feringa*
}

\begin{abstract}
New approaches to achieve control of molecular chirality have enabled the development of methods for asymmetric catalysis and the construction of molecular switches and motors.
\end{abstract}

Keywords: Asymmetric catalysis $\cdot$ Chirality $\cdot$ Molecular motors $\cdot$ Molecular switches

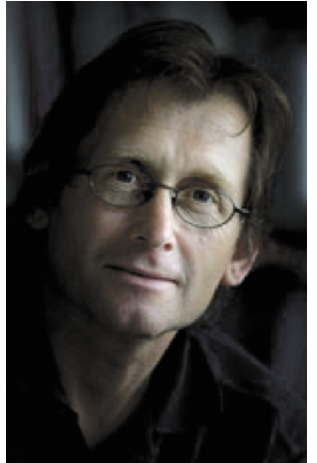

Ben L. Feringa

\section{Introduction}

The unique handedness of the essential molecules of life, the role of stereochemistry in nearly every aspect of our discipline, and the enormous economic implications associated with the production of single enantiomers of pharmaceuticals and other bioactive substances continuously offer major challenges to achieve control of chirality at the molecular, supramolecular and macromolecular level. Chirality is "a signature of life" and is considered to play a decisive role in chembiogenesis [1][2]. The impetus to develop fully catalytic, environmentally benign organic synthesis sets the stage for a new era in asymmetric catalysis [3]. Furthermore, the exploration of funda-

${ }^{\star}$ Correspondence: Prof. B.L. Feringa Stratingh Institute for Chemistry and Chemical Engineering

University of Groningen,

Nijenborgh 4

9747 AG Groningen, The Netherlands

Tel.: +31-50-363 4235/4233/4278

Fax: +31-50-363 4296

E-Mail: b.l.feringa@rug.n mental stereochemical principles provides fascinating opportunities in the emerging areas of smart materials and functional nanosystems [4].

\section{Asymmetric Catalysis}

The conjugate addition of organometallic reagents to $\alpha, \beta$-unsaturated compounds is one of the key carbon-carbon bond forming reactions in organic synthesis (Fig. 1) [5]. The discovery that near-absolute stereocontrol could be obtained for the first time in the copper-catalyzed conjugate addition of organozinc reagents by using phosphoramidites as chiral ligands (Fig. 2) initiated the development of more than a dozen asymmetric transformations based on monodentate ligands [6][7].

The copper-catalyzed conjugate of organozinc reagents has been used to produce a variety of natural products including neurotoxins, prostaglandins, pheromones, and $\beta$-amino acids (Fig. 3). For nearly 30 years it was considered essential to use bidentate chiral ligands in order to achieve high enantioselectivity in asymmetric hydrogenation [3]. In 2000 three groups independently demonstrated that catalysts based on monodentate ligands provide similar levels of stereocontrol [8]. The easy access (often in a one-pot procedure employing cheap starting materials) to phosphoramidites has led to a plethora of new asymmetric hydrogenations and the implementation in the industrial production of a chiral drug intermediate on a multi-ton scale [9]. The modular architecture of monodentate phosphoramidites is an especially attractive feature since it allows rapid optimization of ligand structure for a specific reaction or substrate class, and is compatible with automated synthesis of ligand libraries. This is particularly relevant for the construction of toolkits of chiral catalysts to help chemists meet the short time-frames allowed in route discovery in drug development.

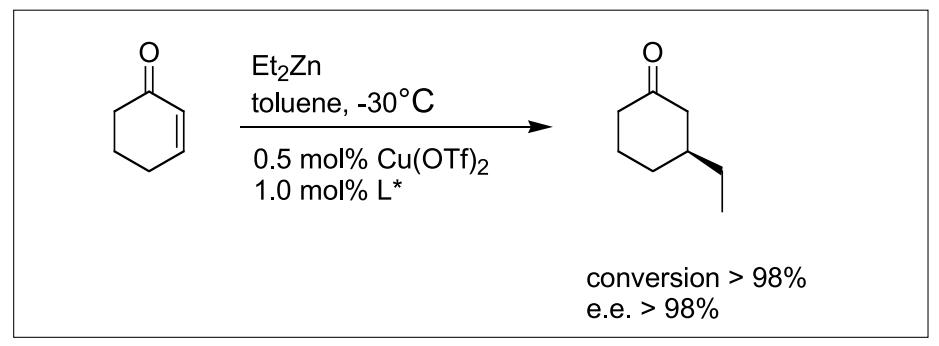

Fig. 1. Catalytic asymmetric conjugate addition of organozinc reagents

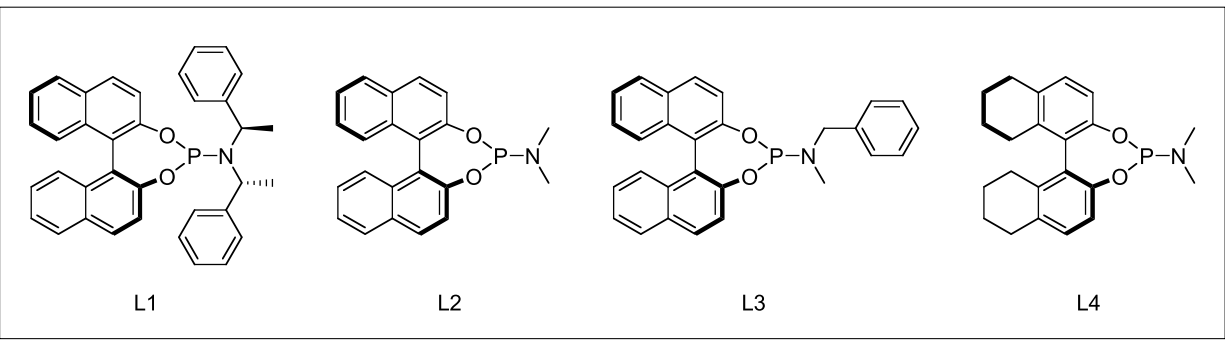

Fig. 2. Representative examples of monodentate chiral phosphoramidite ligands 
<smiles>CC(CN)C(=O)O</smiles><smiles>C=C[14CH2]CCC[C@H](C)CCC[C@H](C)CCCC</smiles><smiles>CCC[C@@H]1CC[C@@H]2[C@@H](C)CCC[C@H]2N1</smiles><smiles>CCCCC[C@H](O)/C=C/[C@@H]1[C@H](CCCCCCC(C)=O)C(=O)C[C@H]1O</smiles>

Fig. 3. Typical examples of bioactive compounds synthesized with a catalytic asymmetric conjugate addition as the key step

$$
\mathrm{M}+\mathrm{P}_{1}^{*}+\mathrm{P}_{2} \longrightarrow \mathrm{P}_{1-\mathrm{M}^{-} \mathrm{P}_{2}}^{\mathrm{P}_{1}^{*} \mathrm{M}^{-} \mathrm{P}_{1}^{*}} \text { hotero-complex }
$$<smiles>[R]/C(=C\c1ccccc1)C(=O)O</smiles>

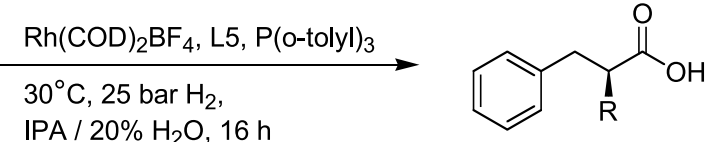

IPA / $20 \% \mathrm{H}_{2} \mathrm{O}, 16 \mathrm{~h}$
$\mathrm{L} 5=$

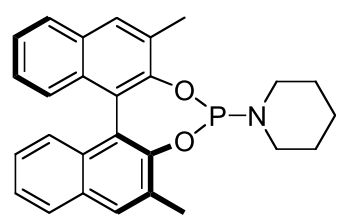

homo-complex (L5): $16 \%$ e.e hetero-compex $\left(\mathrm{L} 5+\mathrm{P}(\mathrm{o} \text {-toly })_{3}\right):>97 \%$ e.e
Fig. 4. The combination of chiral and achiral ligands results in a dramatic increase in enantioselectivity in the preparation of cinnamic acids; key building blocks for renin inhibitors

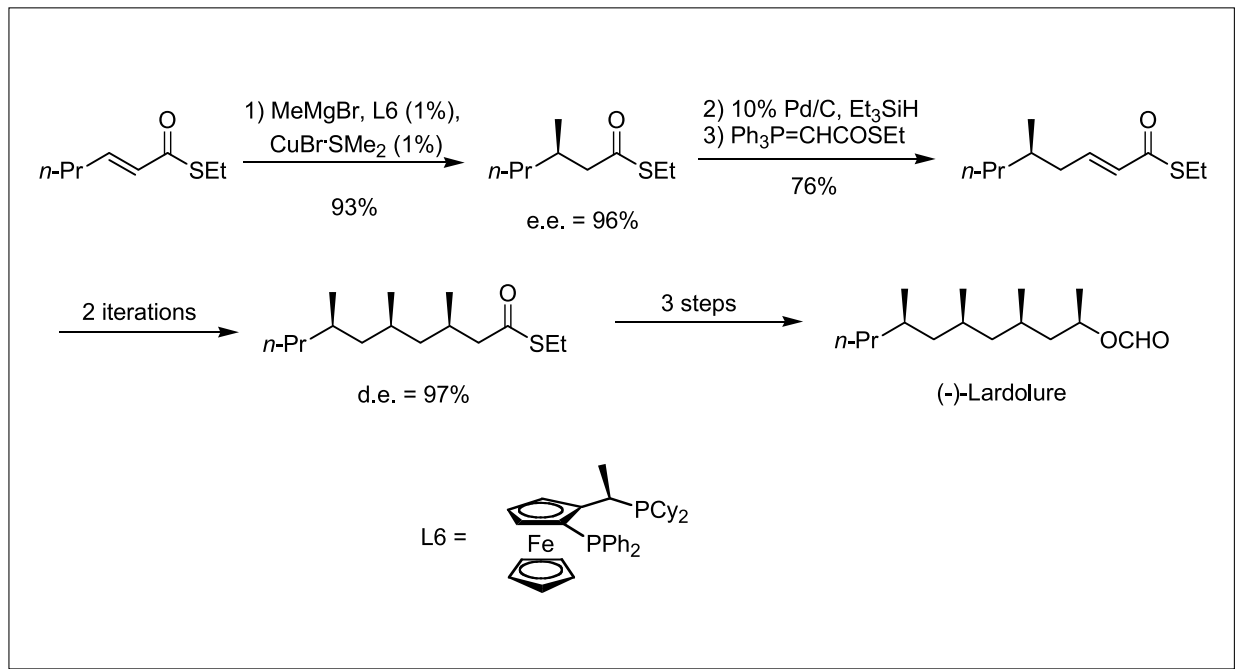

Fig. 5. Synthesis of Lardolure via an iterative introduction of propionate fragments using asymmetric conjugate addition of methylmagnesiumbromide as key step
A special feature of monodentate chiral ligands is the use in mixed-ligand approaches in asymmetric catalysis. Fig. 4 illustrates the spectacular improvement in enantioselectivity that can be reached using a combination of chiral and achiral ligands [10].

In contrast, the breakthrough in the conjugate addition of Grignard reagents was achieved using well-established bidentate phosphine ligands derived from ferrocene (Fig. 5) [11]. With an effective solution for this long-standing problem in asymmetric $\mathrm{C}-\mathrm{C}$ bond formation at hand, iterative catalytic protocols for the construction of oligopropionates were developed to illustrate the potential of this methodology in acyclic stereocontrol.

\section{Molecular Switches and Motors}

Facing the challenges of the nanotechnology era ahead of us it is tempting to predict a decisive role for molecular switches, motors, and nanoscale machines comparable to the introduction of the macroscopic engines and transistors at the dawn of the industrial and information technology revolutions, respectively [12]. The development of molecular switches as potential memory elements for data storage and processing was inspired by the photochemical processes associated with vision. In synthetic chiroptical molecular switches, a light-induced cis-trans isomerization is accompanied by a change in the helicity of the molecule (Fig. 6) [13]. A major advantage of these chiral switches, compared to other photochromic materials used as molecular memory elements, is the possibility for non-destructive read-out using chiroptical techniques [14].

Light-induced switching of molecular structure and helicity has also been used to reversibly address a variety of materials properties. For instance smart gels and liquid crystalline films were developed in which mesoscopic organization, self-assembly, supramolecular chirality or color can be controlled [15].

The incorporation of a photoswitch as a trigger element in a modified mechanosensitive bacterial channel protein allowed the reversible opening and closing of a nanosize valve (Fig. 7) [16]. Reconstitution of the protein in a vesicle membrane allowed the flow of encapsulated molecules from inside the vesicle to be controlled by photoirradiation, constituting a first step toward artificial drug delivery systems using a nanoscale triggering device.

The design and construction of the first light-driven molecular rotary motor provided us with the most extreme stereochemical challenge so far (Fig. 8) [17][18]. Unidirectional rotation of one half of the molecule relative to the other was achieved in four 


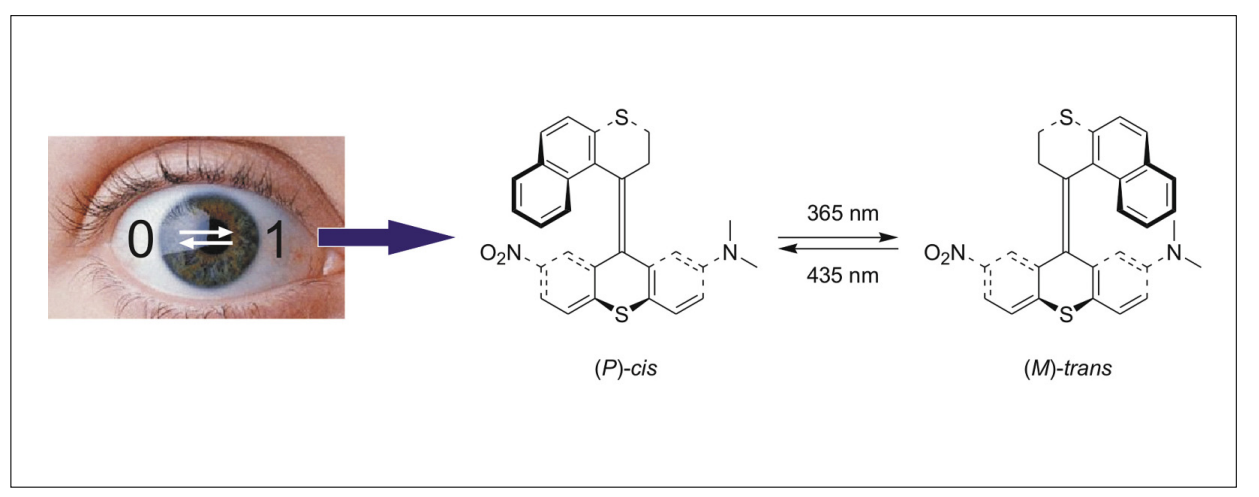

Fig. 6. A chiroptical molecular switch as a binary information storage element. Irradiation at distinct wavelengths results in the interconversion of cis and trans isomers accompanied by helix inversion, allowing each chiral state to be separately addressed.

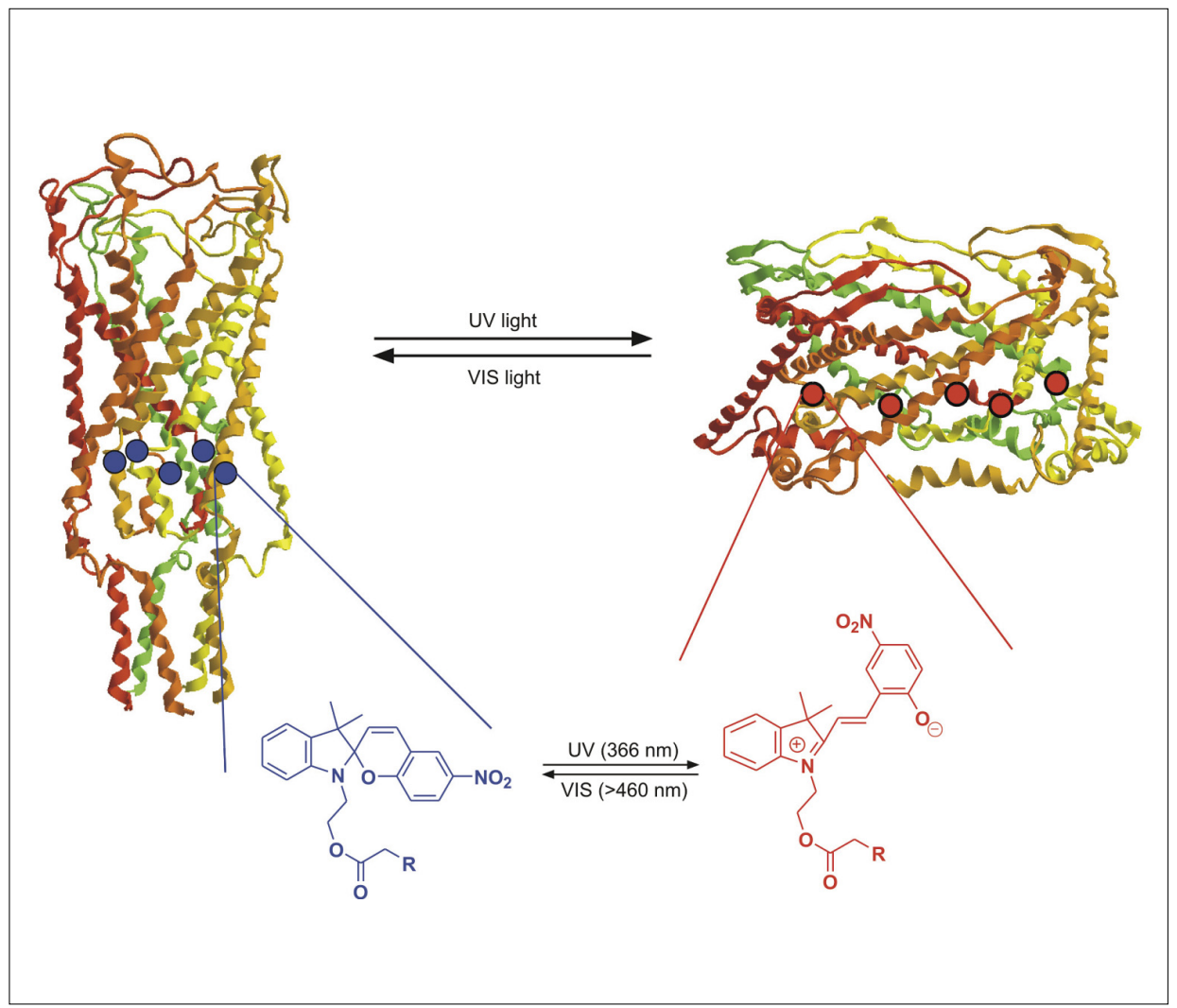

Fig. 7. A nanovalve based on the reversible opening by light of a modified mechanosensitive channel protein

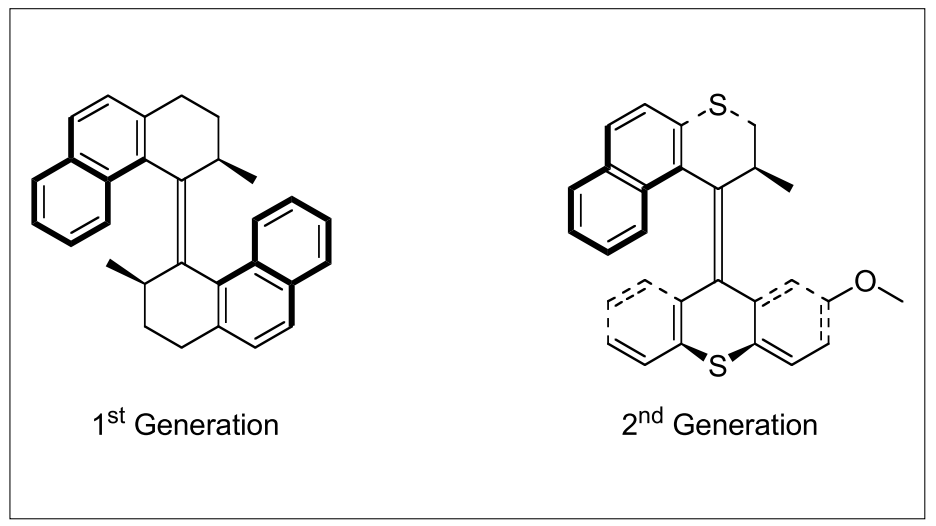

Fig 8. Typical examples of the first and second generation of rotary molecular motors steps, including two photochemical isomerizations each followed by a thermal helix inversion. In the second generation design of molecular motors, the speed of rotation could be increased over a million fold by fine tuning steric and electronic parameters [19].

Through the introduction of two 'legs' on the stator part of the molecule, molecular motors were immobilized on the surface of gold nanoparticles (Fig. 9) [20].

The assembly on surfaces, thereby not compromising the motor function, is a crucial step to be able to build ultimately nanomachines for practical applications and to exploit the coherent motion of a large collection of molecular motors.

Received: January 24, 2006

[1] A. Eschenmoser, Chembiogenesis Symposium, Venice, 2005.

[2] B.L. Feringa, R.A. van Delden, Angew. Chem. Int. Ed. 1999, 38, 3418.

[3] E.N. Jacobsen, A. Pfaltz, H. Yamamoto, 'Comprehensive Asymmetric Catalysis', Vol. 1-3, Springer, Berlin, 1999.

[4] Special issue Scientific American: 'Nanotech, the science of small gets down to business', September 2001.

[5] P. Perlmutter, 'Conjugate Addition Reactions in Organic Synthesis', Pergamon, Oxford, 1992

[6] a) B.L. Feringa, M. Pineschi, L.A. Arnold, R. Imbos, A.H.M. de Vries, Angew. Chem. Int. Ed. 1997, 36, 2620; b) L.A. Arnold, R. Naasz, A.J. Minnaard, B.L. Feringa, J. Am. Chem. Soc. 2001, 123, 5841; c) R. Naasz, L.A. Arnold, A.J. Minnaard, B.L. Feringa, Angew. Chem. Int. Ed. 2001, 40, 927; d) A. Duursma, A.J. Minnaard, B.L. Feringa, $J$. Am. Chem. Soc. 2003, 125, 3700.

[7] For a selection of reviews: a) N. Krause, A. Hoffmann-Röder, Synthesis 2001, 171; b) B.L. Feringa, R. Naasz, R. Imbos, L.A. Arnold 'Modern Organocopper Chemistry', Ed. N. Krause, VCH, Weinheim, 2002, 224; c) B.L. Feringa, Acc. Chem. Res. 2000, 33, 346; d) A. Alexakis, C. Benhaim, Eur. J. Org. Chem. 2002 3221.

[8] a) C. Claver, E. Fernandez, A. Gillon, K. Heslop, D.J. Hyett, A. Martorell, A.G. Orpen, P.G. Pringle, Chem. Comm. 2000 , 961; b) M.T. Reetz, G. Mehler, Angew. Chem. Int. Ed. 2000, 39, 3889; c) M. van den Berg, A.J. Minnaard, E.P.Schudde, J. van Esch, A.H.M. de Vries, J.G. de Vries, B.L. Feringa, J. Am. Chem. Soc. 2000, 122, 11539 .

[9] a) D. Ager, M. van den Berg, A.J. Minnaard, B.L. Feringa, A.H.M. de Vries, C. E. Willans, J.A.F. Boogers, J.G. de Vries, chapter 8 in 'Methodologies in Asymmetric Catalysis', ACS, Washington DC, Ed. S.V. Malhotra, 2004; b) M. van den Berg, D. Pena, A.J. Minnaard, B.L. Feringa, L. Lefort, J.A.F. Boogers, A.H.M. de Vries, J.G. de Vries, Chimica Oggi 2004, Suppl. 


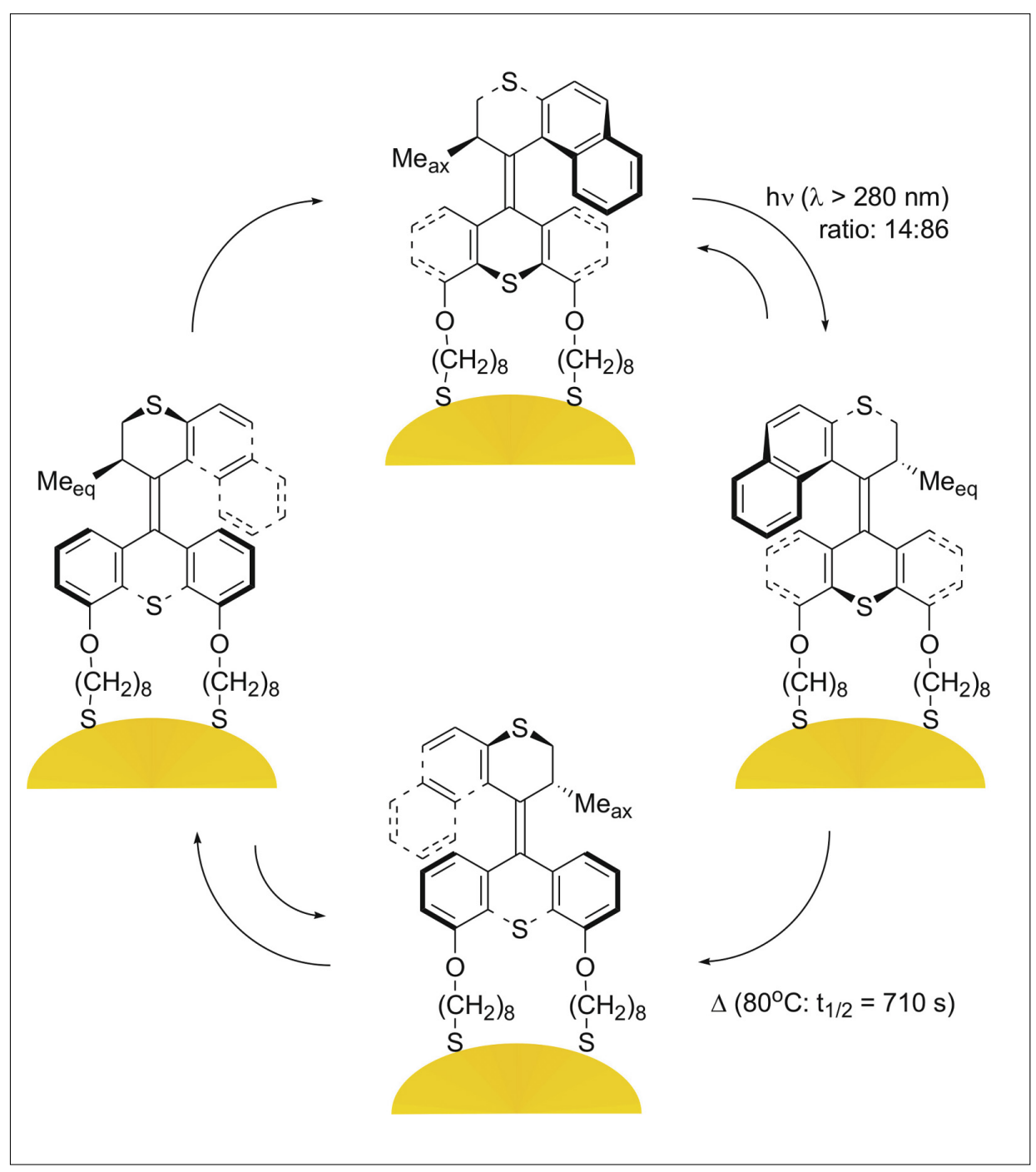

Fig. 9. Unidirectional rotation on gold nanoparticles

'Chiral catalysis - asymmetric hydrogenation'; H. Bernsmann, M. van den Berg, R. Hoen, A.J. Minnaard, G. Mehler, M.T. Reetz, J.G. de Vries, B.L. Feringa, J. Org. Chem. 2005, 70, 943.

[10] R. Hoen, J.A.F. Boogers, H. Bernsmann, A.J. Minnaard, A. Meetsma, Th.D. Tiemersma-Wegman, A.H.M. de Vries, J.G. de Vries, B.L. Feringa, Angew. Chem Int. Ed. 2005, 44, 4209.

[11] a) B.L. Feringa, R. Badorrey, D. Pena Gil, S.R. Harutyunyan, A.J. Minnaard, Proc. Natl. Acad. Sci. USA 2004, 101, 5834; b) F. Lopez, S.R. Harutyunyan, A.J. Minnaard, B.L. Feringa, J. Am. Chem. Soc. 2004, 126, 12784; c) F. Lopez, S.R. Harutyunyan, A. Meetsma, A.J. Minnaard, B.L. Feringa, Angew. Chem. Int. Ed. 2005, 44, 2752; d) R.D. Mazery, M. Pullez, F. Lopez, S.R. Harutyunyan, A.J. Minnaard, B.L. Feringa, J. Am. Chem. Soc. 2005, 127, 9966.

[12] G.M. Whitesides, Scientific American 2001, 285, 70.

[13] 'Molecular Switches', Ed. B.L. Feringa, Wiley-VCH, Weinheim, 2001.

[14] Special issue on 'Photochromism; Memories and Switches', Chem. Rev. 2000, 100, 1683.
[15] a) N.P.M. Huck, W.F. Jager, B. de Lange, B.L. Feringa, Science 1996, 273, 1686; b) R.A. van Delden, N. Koumura, N. Harada, B.L. Feringa, Proc. Natl. Acad. Sci. USA 2002, 99, 4945; c) J.J.D. de Jong, L.N. Lucas, Kellogg, J.H. van Esch, B.L. Feringa, Science 2004, 304, 278.

[16] A. Kocer, M. Walko, W. Meijberg, B.L. Feringa, Science 2005, 309, 755.

[17 N. Koumura, R.W.J. Zijlstra, R.A. van Delden, N. Harada, B.L. Feringa, Nature 1999, 401, 152.

[18] a) 'Molecular Motors', Ed. M. Schliwa, Wiley-VCH, Weinheim, 2003; b) A.R. Pease, J.O. Jeppesen, J.F. Stoddart, Y. Luo, C.P. Collier, J.R. Heath, Acc. Chem. Res. 2001, 34, 433; c) M. Venturi, A. Credi, V. Balzani, 'Molecular Devices and Machines: A Journey into the Nanoworld', Wiley-VCH, Weinheim, 2003; d) J.-P. Collin, C. Dietrich-Buchecker, P. Gavina, M.C. Jiminez-Molero, J.-P. Sauvage, Acc. Chem. Res. 2001, 34, 477; B.L. Feringa, Acc. Chem. Res. 2001, 34, 504; e) T.R. Kelly, H. De Silva, R.A. Silva, $\mathrm{Na}$ ture 1999, 401, 150; f) D.A. Leigh, J.K.Y. Wong, F. Dehez, F. Zerbetto, Nature 2003, 424, 174; g) T. Hugel, N.B. Holland, A.
Cattani, L. Moroder, M. Seitz, H.E. Gaub, Science 2002, 296, 1103; h) S.P. Fletcher, F. Dumur, M.M. Pollard, B.L. Feringa, Science 2005, 310, 80.

[19] a) D. Pijper, R.A. van Delden, A. Meetsma, B.L. Feringa, J. Am. Chem. Soc. 2005, 127, 17612; b) J. Vicario, A. Meetsma, B.L. Feringa, Chem. Comm. 2005, 5910.

[20] R.A. van Delden, M.K.J. ter Wiel, M.M. Pollard, J. Vicario, N. Koumura, B.L. Feringa, Nature 2005, 437, 1337. 